vacant niche by providing a comprehensive, up-to-date and accessible introduction to Earth-science applications of modern theories in rock physics. The authors lay the groundwork by developing the basic description of the microstructures of porous and heterogeneous rocks. They discuss developments in chemistry and physics applicable to rock physics, such as surface adsorption of ions, effective medium theories for calculating bulk properties of heterogeneous and porous media, and fractal descriptions of fractures and pore surfaces, and then use these concepts to discuss acoustic properties, electrical conductivity, dielectric properties, thermal conductivity and magnetic properties of bulk rocks, particularly as influenced by fluids.

Two features set the book apart from others. First, it is comprehensive. The chapters on electrical conductivity, dielectric properties, thermal conductivity and magnetic properties, as well as the more traditional sections on the mechanical properties of rocks and flow through porous media, make this the only text to cover virtually all of the physical prop-

erties that concern rock physicists. Second, the text is well integrated, with concepts introduced early on in one context reapplied later in another. For example, effective medium theory is introduced in the discussion of elastic properties of heterogeneous media, and then reappears in chapters covering electrical, dielectric and thermal conductivity. Students will find this continuity helpful and enlightening.

The book is a translation of a volume originally published in French, Introduction à la Physique des Roches (Hermann Éditeurs des Sciences et des Arts, Paris, 1992). It has abundant references to recent work (up to about 1990), a feature that will enable readers to have good access to the current literature.

It will be useful for a rigorous graduate course on the physical properties of rocks, as well as being valuable as a reference for professionals interested in the subject.

James A. Tyburczy is in the Department of Geology, Arizona State University, Box 871404, Tempe, Arizona 85287-1404, USA.

\title{
Solving the monkey puzzle
}

\section{Andrew Whiten}

Self-Awareness in Animals and Humans. Edited by Sue Taylor Parker, Robert W. Mitchell and Maria L. Boccia. Cambridge University Press: 1994. Pp. 442. £40, $\$ 59.95$.

WHAT do you see when you look in the mirror? Why, your self, of course, you will be thinking to yourself. But here are two quite different senses of 'self' - the self we see reflected and the self who reflects. Our sense of self is so central to us that questions about the emergence of such forms of self-awareness in evolution or development could hardly be more fundamental. And if answers can be provided, they may well be seen as relevant to our codes of morality concerning, for example, the ethical treatment of animals.

One of the most influential steps towards a scientific approach to such apparently ineffable issues came in the 1970s, when Gordon Gallup showed that chimpanzees and orangutans would not only use mirrors to inspect themselves, but would wipe off marks applied to their faces under anaesthetic; yet monkeys failed to do these things. Gallup suggested that the apes alone, like humans, were showing an awareness of self, from which further capabilities could arise, such as intentional deception and empathy.

Nearly all of the 16 'animal' chapters in this book are about these responses to mirrors: to accept the title as most people would read it, one has to buy Gallup's generous interpretation. But if one accepts this focus, the volume offers a comprehensive and indispensable survey of the state of the art, presented by today's principal workers and including interesting extensions of the approach to other taxa.
Coincidentally, Celia Heyes (Animal Behaviour 47, 909; 1994) has argued (with some justification, in my view) that owing to the lack of proper controls, the Gallup mark-test does not in fact show evidence of mirror-use. The obvious control procedure of, say, simultaneously shammarking the chin when paint is applied to the forehead, has been neglected. Ironically, something similar to this procedure is here reported with great effect in a gorilla, the one great ape species hitherto dismissed as lacking self-recognition. Together with spontaneous mirror use, this evidence is compelling - much more so than that presented for monkeys and dolphins. Although a monkey seems to see another monkey in its reflection, it is beginning to look as if, in the case of all four genera, a great ape may in some sense see itself (although not all do, and Daniel Povinelli suggests an interesting explanation).

But just because apes can see their self reflected does not necessarily imply the other sense - that they self-reflect. When it comes to the psychological meaning of these patterns, nobody knows. The book does show that theory is developing, some at least in the direction of testable predictions. The integration of findings from developmental, abnormal and comparative psychology is already used in this spirit by several authors. Scarcely a stone is left unturned in laying out the theoretical options, and particular credit is due both to Parker and to Mitchell for their efforts to this end.

Andrew Whiten is in the School of Psychology, University of St Andrews, St Andrews, Fife KY16 9JU, UK.

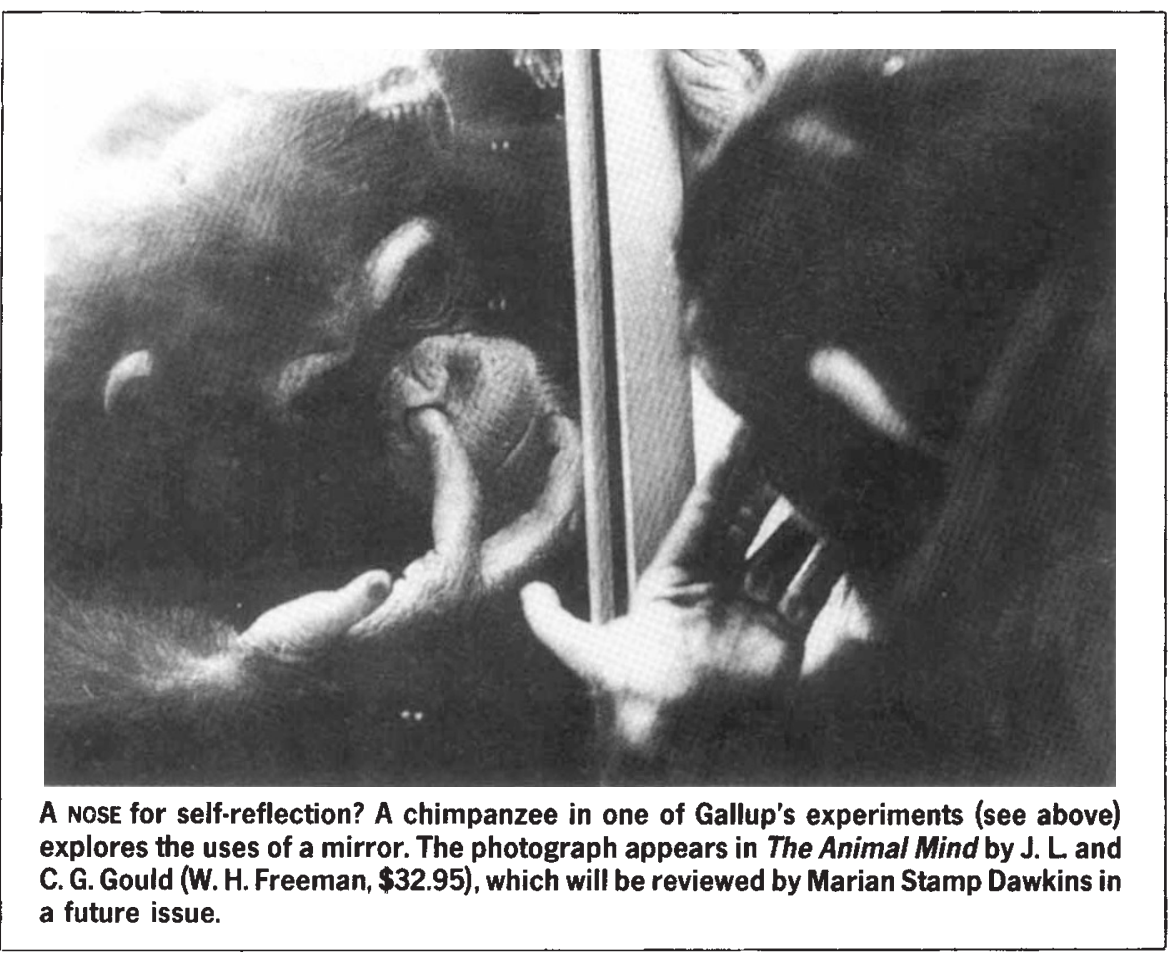

